\title{
The effect of apparent movement on mental rotation
}

\author{
MICHAEL C. CORBALLIS \\ University of Auckland, Auckland, New Zealand \\ and \\ A. ROGER BLACKMAN \\ Simon Fraser University, Burnaby, British Columbia, Canada
}

\begin{abstract}
Eleven subjects were timed as they judged whether a small bar perpendicular to one side of a clockhand would point left or right if the hand was pointing upward (i.e., at the "12 o'clock" position). The clockhand was shown in two successive orientations $30^{\circ}$ apart, so that it was perceived to jump from one to the other in either a clockwise or a counterclockwise direction. Reac. tion times were consistent with the interpretation that the subjects "mentally rotated" the clockhand from its perceived orientation back to the upright before making their decisions. The direction of the jump influenced perceived orientation but did not influence either the direction or rate of mental rotation itself.
\end{abstract}

Under appropriate conditions, the presentation of a shape in different, successive locations or orientations can give rise to apparent movement. Shepard (1984) has argued that the path of apparent movement is constrained by an internalized kinematic geometry, or a built-in knowledge of how things actually move in the real world. One example of this is representational momentum, in which the last-perceived orientation of a shape is extrapolated in the direction of an apparent rotation. It is as though the momentum of the shape has carried it beyond the point where it actually appeared (Freyd \& Finke, 1984).

Freyd (1987) has suggested that representational momentum is distinct from mental rotation, in which a person may imagine a shape, presented in one orientation only, rotating to some different orientation (e.g., Cooper \& Shepard, 1973). Freyd notes that the temporal parameters of the two phenomena are different, and that representational momentum is a mandatory, "cognitively impenetrable" process, whereas mental rotation is a voluntary process. The internal mechanisms underlying apparent motion in general also may be different from mental rotation in these respects, although similar in other respects, such as analog representation.

In the present study, we examine the influence of apparent rotation on mental rotation. Earlier experiments have shown that the mental rotation of tilted letters to their upright orientations may be influenced by a rotation aftereffect, induced by having subjects watch a rotating disk before being exposed to each letter (Corballis, 1986; Cor-

The authors wish to thank Lynette J. Tippett for her assistance in the design and execution of this study. Correspondence may be addressed to A. Roger Blackman, Department of Psychology, Simon Fraser University, Burnaby, British Columbia V5A 1S6, Canada. ballis \& McLaren, 1982). Specifically, mental rotation appears to be slowed by an aftereffect in the direction opposite to the shortest angular route to the upright, whereas it appears to be speeded by an aftereffect in the same direction as the shortest angular route (Corballis, 1986). However, this may not be a direct effect on rotation rate itself, but may be an influence on the direction of rotation. That is, if the aftereffect opposes rotation through the shortest angular distance to the canonical orientation, subjects may be induced on at least some trials to rotate "the long way around." For example, if a stimulus is tilted $120^{\circ}$ from the upright, the shortest distance back to the upright would of course be $120^{\circ}$, but an aftereffect might induce the subject to rotate the stimulus through the larger angle of $240^{\circ}$ to the upright.

We might, however, expect the influence of apparent rotation to be different from that of a rotation aftereffect. The rotation aftereffect has the paradoxical property that it causes a shape to appear to rotate without changing its orientation, whereas apparent rotation actually depends on a change in orientation. Consequently, we might expect apparent rotation to influence mental rotation by inducing changes in the perceived orientation of the stimulus, perhaps through representational momentum. We wanted also to determine whether apparent rotation would affect the rate and/or direction of mental rotation.

The stimulus was a single hand on a clockface, with a small bar halfway along it and perpendicular to one side of it. The subject's task was to decide whether the bar pointed left or right when the hand was in the upright (" 12 o'clock") position-a mirror-image discrimination that we assumed would require mental rotation (see Corballis, 1988b; Takano, 1989). However, the hand was shown in two consecutive positions $30^{\circ}$ apart (e.g., at " 4 o'clock," 
then at "5 o'clock"), so that it was seen to jump from one to the other. The question was whether or not this jump would influence mental rotation of the hand to the upright.

\section{METHOD}

\section{Subjects}

The subjects were 4 men and 7 women, drawn from a third-year undergraduate course in cognitive psychology.

\section{Apparatus and Stimuli}

The stimuli were generated by an Apple Ile computer and presented on a fast-phosphor magenta screen. Stimulus presentations were synchronized with the 50-cps frame rate according to the procedures described by Cavanagh and Anstis (1980).

The stimuli were presented against a clockface represented by 12 dots arranged in a circle at $30^{\circ}$ intervals (i.e., marking the positions of the hours), with a radius of $30 \mathrm{~mm}$. The stimuli were constructed as follows: A line was drawn from the center of the clockface to one of the dots to represent a hand on the clockface. A small bar, $5 \mathrm{~mm}$ long, was drawn perpendicular to one side of this line, halfway along it. This stimulus was presented for seven frames, or approximately $140 \mathrm{msec}$, in one orientation, and then for another seven frames in a neighboring orientation, with no intervening interval. This gave a clear perceptual impression of rotation, so that the stimulus seemed to jump $30^{\circ}$ (or one hour on the clockface). The screen then went blank.

There were 48 different stimuli, made up as follows: First, the clockface was divided into $1230^{\circ}$ sectors, which were numbered in clockwise steps from the top (i.e., the first sector was between 12 o'clock and 1 o'clock, the second between 1 o'clock and 2 o'clock, and so on). Each sector was swept by either a clockwise or a counterclockwise jump. For each jump, the bar was either to the left or to the right of the line relative to its upright orientation. In a given block of trials, each of these conditions was presented twice, for a total of 96 trials, which were randomly ordered.

\section{The Task}

The subject was required to press the $\mathrm{N}$ key if the bar would be was to the right of the line if the line was upright (i.e., in the 12 o'clock position), and the B key if it would be to the left of the line. These keys are adjacent on the bottom row of the console, and the labels LEFT and RIGHT were affixed to the space bar immediately below them. The subjects were instructed to respond as quickly as possible to the appearance of the stimulus; the direction of the jump was irrelevant to the decision. Reaction times (RTs) were measured from stimulus offset.

\section{Procedure}

Each subject was given two blocks of 96 experimental trials, with each block preceded by 10 practice trials in which the stimuli were chosen at random from the $\mathbf{4 8}$ possible stimuli. Before each trial, the subject sat with the index finger of each hand resting lightly on the appropriate response keys. The subject initiated stimulus presentation by pressing either key. The stimulus appeared $500 \mathrm{msec}$ after the press. If the subject made an error, that stimulus was repeated at the end of the block.

\section{RESULTS}

In analyzing the results, orientations were classified by sector, measured clockwise from the upright, as described above.

\section{Errors}

The subjects made errors on $9.2 \%$ of the trials. The errors were subjected to an analysis of variance, in which the independent variables were orientation, block, direction of the jump, and direction of the bar. The only significant main effect was that of orientation $[F(11,110)=$ 5.06, $M S_{\mathrm{e}}=.4451, p<.005$ ], with errors at a minimum in the second sector (1.1\%) and rising to a maximum in the eighth $(23.3 \%)$.

\section{Reaction Times}

RTs for correct responses were subjected to an analysis of variance with the same independent variables as those in the analysis of errors.

There was a highly significant main effect of orientation $\left[F(11,110)=20.15, M S_{\mathrm{e}}=103,439, p<.001\right]$, as well as a significant interaction between orientation and direction of the jump $\left[F(11,110)=5.35, M S_{\mathrm{e}}=38,634\right.$, $p<.001]$. Mean RTs for each sector and direction of jump are plotted in Figure 1.

It is clear that RT is a sharply increasing function of orientation, rising to a maximum in the 6th sector for clockwise jumps and in the 7th sector for counterclockwise jumps-that is, the functions are phase-shifted relative to one another by about one sector. This might be simply explained in terms of differences in the perceived locations of the jumps, depending on their directions.

To explore this idea, we assumed that the subjects mentally rotated the hand back to the upright, or 12 o'clock orientation, before making their judgments. RT should therefore be a linear function of angular distance of the perceived location of the hand from the upright. We then computed the angular adjustment to each sector that maximized the fit of the actual RTs to the predicted RTs. Our computational procedures are available on request.

For clockwise jumps, the fit was maximized when the adjustment was $20^{\circ}$ clockwise from the beginning of each sector-that is, when a jump through the first sector was actually perceived at $20^{\circ}$ from the upright. This fit accounted for $93.95 \%$ of the variance; the slope of the function yielded an estimated mental-rotation rate of $347^{\circ}$ per second, which is very close to that typically found in experiments on mental rotation (e.g., Cooper \& Shepard, 1973; Corballis, 1982). For counterclockwise jumps, the adjustment was $38^{\circ}$ counterclockwise from the beginning of each sector, so that, according to this analysis, a jump through the first sector was perceived at $352^{\circ}$ clockwise from the upright (or $8^{\circ}$ counterclockwise from the upright). This fit accounted for $93.22 \%$ of the variance and yielded an estimated mental-rotation rate of $344^{\circ}$ per second. Note that this adjustment for counterclockwise jumps suggests a slight extrapolation $\left(8^{\circ}\right)$ beyond the actual sector, which may be attributed to representational momentum (see Freyd \& Finke, 1984). Perhaps the subjects tended to "predict" the stopping point on the assumption that the jumps were going to be clockwise, thus les- 


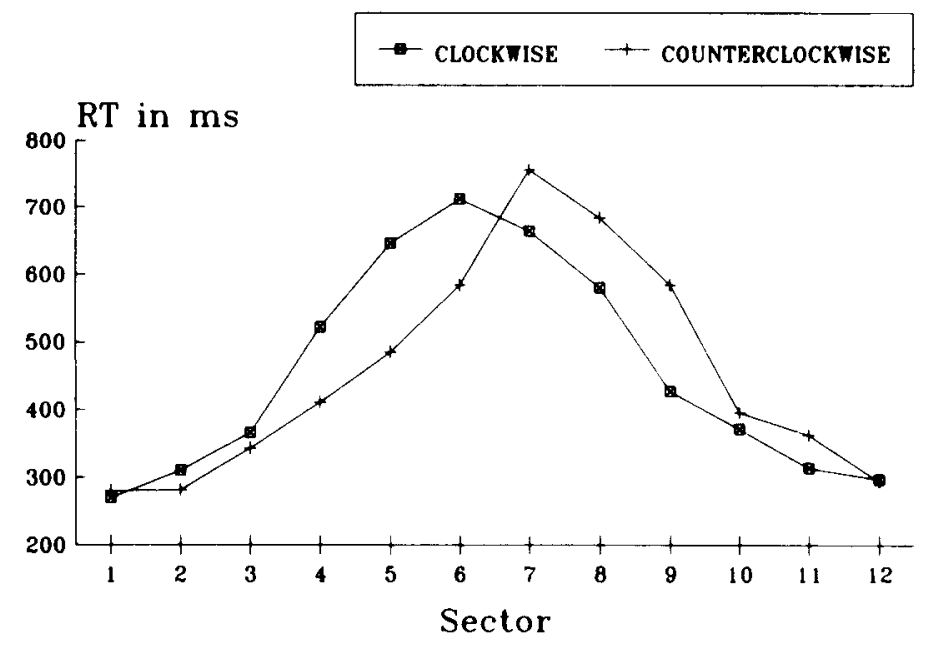

Figure 1. Mean RTs in milliseconds following clockwise or counterclockwise apparent motion as a function of stimulus orientation (sector). Numbered sectors denote successive $30^{\circ}$ sweeps: Sector $1=0^{\circ}-30^{\circ}$; Sector $2=30^{\circ}-60^{\circ}$, and so forth.

sening the effect of representational momentum for the more familiar clockwise direction.

These data are therefore highly consistent with the notion of mental rotation and suggest that the subjects mentally rotated the hand from its perceived location by the shortest route to the upright; there was little evidence that they rotated "the long way around" or that the rate of rotation was influenced by the direction of the jump.

\section{Fits to Individual Data}

Since individual subjects might have differed in the perceived orientations of the stimulus prior to mental rotation, angular adjustments to the functions, as described above, were computed for each subject. The resulting parameters are summarized in Table 1.

These parameters are compatible with mental rotation in virtually every case. The estimated rates are all within expected bounds, and the variances accounted for are reasonably high. The mean estimates of mental-rotation rate are $384^{\circ}$ per second for clockwise jumps and $405^{\circ}$ per second for counterclockwise jumps, again close to the values obtained in previous research. Cooper and Shepard (1973) reported estimates of individual rates ranging from $164^{\circ}$ per second to $800^{\circ}$ per second, and only 3 of the 22 rates we computed fell outside this range, in each case only by a fairly narrow margin.

Of the 22 estimated angular adjustments, 12 lay within the jump, indicating that the stimulus was perceived somewhere between the two presented orientations. Nine lay beyond the jump, implying an effect of representational momentum; this was most marked in the case of Subject 11 . In one case (Subject 4 , clockwise jumps), the perceived location was $4^{\circ}$ behind the starting location, but this might have been due to an error of estimate or perhaps to consistent head tilt.

\section{Analysis of Adjusted Functions}

The sectors of each jump, for each subject and each direction of jump, were relabeled according to the adjustments computed in Table 1. These adjustments can be taken to indicate the perceived location of each jump, on the assumption that the subjects rotated to the upright. Thus, the sector of the jump that was perceived to lie closest to the upright was relabeled as the 1 st sector, the next as the 2nd sector, and so on. For Subject 2, for example, the adjustment for clockwise jumps was $+35^{\circ}$, which may be interpreted to mean that jumps through the 12 th sector were perceived as falling $5^{\circ}$ into the $1 \mathrm{st}$ sector. The 12 th sector was therefore relabeled as the $1 \mathrm{st}$, the $1 \mathrm{st}$ as the 2 nd, and so on. This relabeling has the effect of maximizing the fits of RTs to an idealized mentalrotation function, in which RT rises linearly from the 1st

Tuble 1

Estimated Parameters Obtained by Fitting Idealized Mental-Rotation Functions to RTs for Each Subject

\begin{tabular}{cccccccc}
\hline & \multicolumn{3}{c}{ Clockwise Jumps } & \multicolumn{3}{c}{ Counterclockwise Jumps } \\
\cline { 2 - 4 } \cline { 6 - 7 } Subject & Adjustment & VAF & Rate & Adjustment & VAF & Rate \\
\hline 1 & +24 & 79.08 & 302 & -19 & 63.11 & 520 \\
2 & +35 & 77.79 & 404 & -56 & 95.42 & 342 \\
3 & +45 & 67.46 & 280 & -30 & 82.51 & 348 \\
4 & -4 & 82.83 & 139 & -45 & 72.36 & 150 \\
5 & +29 & 74.94 & 493 & -39 & 76.65 & 339 \\
6 & +11 & 83.33 & 552 & -22 & 75.86 & 478 \\
7 & +22 & 91.53 & 255 & -38 & 81.34 & 228 \\
8 & +42 & 67.33 & 600 & -27 & 67.48 & 436 \\
9 & +3 & 74.05 & 503 & -11 & 81.13 & 949 \\
10 & +11 & 83.34 & 259 & -25 & 75.83 & 324 \\
11 & +74 & 84.60 & 435 & -68 & 90.82 & 345 \\
\hline
\end{tabular}

Note-Adjustment was measured in degrees from the beginning of each jump, with positive scores representing clockwise and negative scores representing counterclockwise. $\mathrm{VAF}=$ percent variance accounted for . Rates are given in degrees per second. 


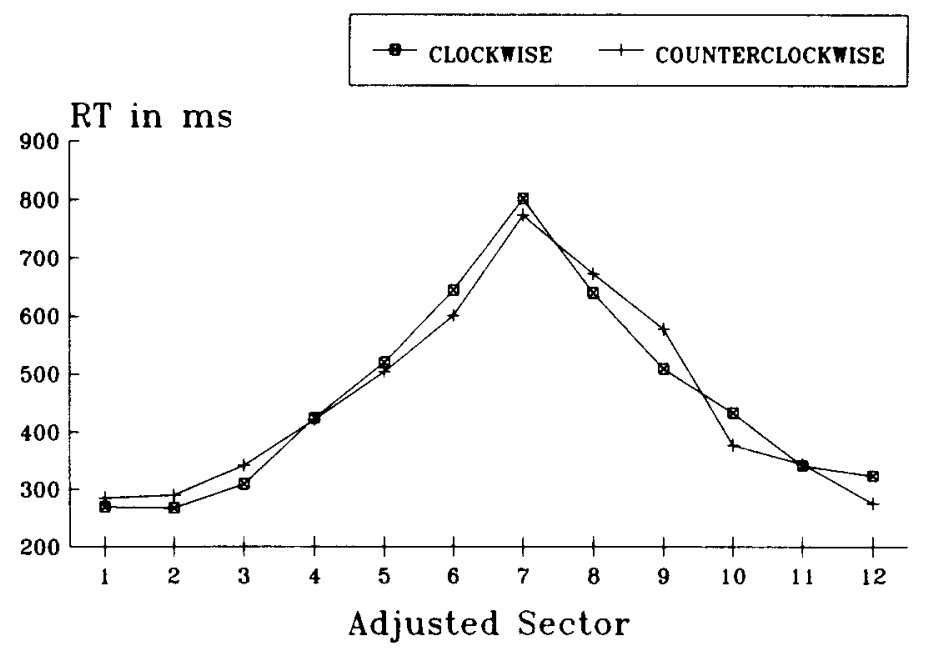

Figure 2. Mean RTs in milliseconds following clockwise or counterclockwise apparent motion as a function of adjusted stimulus orientation (sector). See text for the procedures used to adjust the data.

to the 7th sector and then falls linearly from the 7th to the 12th. With this adjustment to each subject's data, the RT functions are effectively aligned to within $15^{\circ}$.

An analysis of variance of the adjusted RTs removed the significant interaction between orientation and direction of the jump $\left[F(11,330)=1.54, M S_{\mathrm{e}}=6,842, \mathrm{n} . \mathrm{s}\right]$. The main effect of orientation remained highly significant $\left[F(11,330)=40.26, M S_{\mathrm{e}}=13,868, p<.001\right]$, and corresponded to an estimated rotation rate of $343^{\circ}$ per second.

Figure 2 shows the mean RTs for each direction of jump plotted as a function of the renumbered sectors. Note that the functions are now effectively superimposed and resemble the classic mental-rotation functions reported by Cooper and Shepard (1973).

\section{DISCUSSION}

The data were consistent with the proposition that the subjects mentally rotated the hand from its perceived orientation to the upright by the shortest angular route. However, the perceived orientation was itself somewhat influenced by the jump, and, in the case of some subjects, it was extrapolated beyond the end of the jump, presumably as a consequence of representational momentum (Freyd \& Finke, 1984). The fact that the perceived orientation lay within the jump sector for other subjects does not necessarily mean, of course, that these individuals were not subject to representational momentum. The subjects were not required to mentally rotate from the "last seen" orientation, but they might have coded the stimulus at any position along its perceived path. Only rarely did the perceived orientation lie close to either of the orientations in which the stimulus was actually presented.
When the functions for clockwise and counterclockwise jumps were corrected for individual variations in this perceived orientation, they were almost exactly superimposed (see Figure 2) and closely resembled the symmetrical, peaked function reported in earlier experiments on mental rotation (e.g., Cooper \& Shepard, 1973). On these grounds, we conclude that the direction of the jump did not influence either the rate or the direction of mental rotation. In this regard, the effect of apparent motion is not like that of a rotation aftereffect (cf. Corballis, 1986; Corballis \& McLaren, 1982).

The results may also be contrasted with those of a similar study by Corballis (1988a), in which subjects were required to judge the actual direction of a $30^{\circ}$ jump of a hand on a clockface; the stimuli were the same as those of the present study, except that the bar was not present. When the subjects were required to judge whether the jump was clockwise or counterclockwise, they seldom used a mental-rotation strategy, suggesting that discrimination of clockwise from counterclockwise may be an exception to the general rule that mirror-image discriminations require mental rotation to some canonical orientation (for speculations as to why this might be so, see Corballis, 1988b, and Takano, 1989). However, when they were required to judge whether the jump would move left or right past the 6 o'clock position if allowed to continue in the same direction, the subjects more frequently used a mental-rotation strategy. The direction of mental rotation, however, was strongly influenced by the direction of the jump. That is, subjects often rotated the stimulus to the 6 o'clock position in the direction of the jump, even if this meant "going the long way around."

This last result is perhaps not surprising, since the direction of the jump was critical to the decision itself and ro- 
tation in the direction of the jump was to some extent implicit in the instructions. There was no evidence that the direction of the jump influenced the direction of mental rotation in the present study, in which the jump was irrelevant to the decision.

In summary, the most parsimonious interpretation of the evidence to date is that apparent motion may influence mental rotation in two ways. First, it may influence the perceived orientation of the stimulus, and so influence how long it will take to rotate the stimulus to the upright. However, this does not apply in the case of a rotation aftereffect, which does not influence perceived orientation. Second, it may influence the direction in which subjects rotate a stimulus to the upright, as in the studies by Corballis and McLaren (1982), Corballis (1986), and Corballis (1988a). There is still no compelling evidence that apparent motion can influence the rate of mental rotation. As Freyd (1987) implies, then, the actual mechanisms of perceived movement may be rather different from those of imagined movement.

\section{REFERENCES}

Cavanagh, J. P., \& Anstis, S. M. (1980). Visual psychophysics on the Apple II: Getting started. Behavioral Research Methods \& Instrumentation, 12, 614-626.
Cooper, L. A., \& Shepard, R. N. (1973). Chronometric studies of the rotation of mental images. In W. G. Chase (Ed.), Visual information processing (pp. 75-176). New York: Academic Press.

Corballis, M. C. (1982). Mental rotation: Anatomy of a paradigm. In M. Potegal (Ed.), Spatial abilities: Developmental and physiological foundations (pp. 173-198). New York: Academic Press.

Corballis, M. C. (1986). On imagined revolutions. In D. F. Marks (Ed.), Theories of image formation (pp. 151-168). New York: Brandon House.

Corballus, M. C. (1988a). Distinguishing clockwise from counterclockwise: Does it require mental rotation? Memory \& Cognition, 16, 567-578

Corballis, M. C. (1988b). Recognition of disoriented shapes. Psychological Review, 95, 115-123.

Corballis, M. C., \& McLaren, R. (1982). Interaction between perceived and imagined rotation. Journal of Experimental Psychology: Human Perception \& Performance, 8, 215-224.

Freyd, J. J. (1987). Dynamic mental representations. Psychological Review, 94, 427-438.

FREYD, J. J., \& FINKE, R. A. (1984). Representational momentum. Journal of Experimental Psychology: Learning, Memory, \& Cognition, 10, 126-132.

SHEPARD, R. N. (1984). Ecological constraints on internal representations: Resonant kinematics of perceiving, imagining, thinking, and dreaming. Psychological Review, 91, 417-430.

Takano, Y. (1989). Perception of rotated forms: A theory of information types. Cognitive Psychology, 21, 1-59.

(Manuscript received February 24, 1989; revision accepted for publication March 7, 1990.) 